\title{
Nitrogen-Doped Carbonized Polymer Dots (CPDs) and their Optical and Antibacterial Characteristics: A Short Review
}

\author{
Biya Elsa Saji ${ }^{1}$, Midhun Saji ${ }^{1}$, Neethu Joseph ${ }^{1}{ }^{(\mathbb{D})}$, Manoj Balachandran ${ }^{2, *(\mathbb{D})}$ \\ 1 Department of Physics \& Electronics, CHRIST (Deemed to be University), Bangalore, India; \\ neethu.joseph@res.christuniversity.in $\quad$ (N.J.); $\quad$ biya.saji@phy.christuniversity.in $\quad$ (B.E.S.); \\ midhun.saji@phy.christuniversity.in (M.S.); \\ 2 Department of Physics \& Electronics, CHRIST (Deemed to be University), Bangalore, India; manoj.b@christuniversity.in; \\ * Correspondence: manoj.b@ christuniversity.in (M.B.); neethu.joseph@ res.christuniversity.in (N.J.);
}

Scopus Author ID 36626120900

Received: 22.07.2021; Revised: 18.09.2021; Accepted: 22.09.2021; Published: 16.10.2021

\begin{abstract}
Substantial advancements in the field of Carbon Dots (CDs) and their derivatives in recent years can be accredited to their tunable properties. Recently Carbonized Polymer Dots (CPDs) are the emerging form in the CDs family, which possesses a typical polymer/Carbon hybrid structure and properties due to its incomplete carbonization. Alteration of various parameters during the synthesis process suggested that the properties of CPDs depend on temperature and $\mathrm{pH}$. It was found that doping of CPDs using nitrogen enhanced its optical properties, thereby being used as biomarkers. CPDs generally hold a strong green and blue emission, while intense red luminescence was observed doping with nitrogen. Photoluminescence Quantum Yield (PLQY) was also found to increase with the increase in doping and temperature. Doped CPDs find several applications, including bio-imaging, LEDs, etc. In this review, we focus on analyzing the increase in efficiency of CPDs with the process of doping considering optical and antibacterial applications.
\end{abstract}

Keywords: Carbonized Polymer Dots (CPDs); fluorescence; nitrogen doping; antibacterial studies.

(C) 2021 by the authors. This article is an open-access article distributed under the terms and conditions of the Creative

Commons Attribution (CC BY) license (https://creativecommons.org/licenses/by/4.0/)

\section{Introduction}

Carbon Dots (CDs), highly fluorescent carbon-based nanomaterial, having great significance due to their remarkable optical properties like bright luminescence, higher absorption, exceptional light stability, and resistance to light bleaching [1], lesser toxicity [2], good biocompatibility [3], environment-friendly [4], and its easy preparation [5]. They have wide applications in numerous fields such as biomedicine, anticounterfeiting, catalysis, LEDs, photovoltaic devices, sensing, etc. [6]. Synthesis of CDs can be done by both the top-down and bottom-up methods. In the bottom-up approach, a hybrid structure of CDs with polymer and carbon specifications can be established through hydrothermal routes $[7,8]$. These are called the Carbonized Polymer Dots (CPDs). This new material finds a wide range of applications in different fields by using its superior optical, electrochemical properties and excellent biocompatibility.

CPDs have a carbon/polymer hybrid structure with a low carbonization degree comprising a carbon core and, on the surface, large functional groups/polymer chains $[9,10]$. CPDs are generally formed as a part of partial carbonization of the polymer cluster [6]. The carbon core is distributed into four types; two types of fully carbonized cores comparable to 
the Carbon Nano Dots (CNDs) [11], a para-crystalline carbon structure composed of small carbon clusters enclosed by polymer frames [11,12], and highly dehydrated cross-linking and close-knit polymer frame structure [7, 13]. CPDs possess unique characteristics, like high quantum yield, large oxygen content, and exceptional aqueous solubility. Characterization data exhibited the presence of a significant amount of oxygen and nitrogen in addition to carbon core [8]. CPDs possess better stability than polymers due to carbonization and better compatibility when compared with Quantum Dots (QDs) due to the presence of polymer chains. CPDs combines the advantages of both polymers and QDs and are environment being lesser cost and non-toxic[14]. Carbon, being the main element, makes CPDs non-toxic and metabolizable compared with metal elements. The facile synthesis methods such as hydrothermal, solvothermal, or microwave-assisted methods are proven to be effective.

The processes involved in the synthesis of CPDs are dehydration, assembly, crosslinking, and carbonization. CDs undergo condensation and cross-linking to create polymer clusters and later undergo dehydration and carbonization to reach CPDs [3-10,15-17]. An intermediate product developed during the process of CD's synthesis is polymer-like clusters [16]. As the reaction progresses, partial carbonization may occur in these clusters, producing polymer/carbon hybrid structures rather than pure carbon structures. PL behavior of CPDs is influenced by both surface functional groups and carbon cores since both can significantly alter the electronic structure. The presence of ample surface groups makes CPDs hydrophilic enough without additional alteration, advantageous to application in living organisms [15]. Nitrogendoped CPDs showed intense red luminescence due to effective hydrogen bonds and aromatic $\pi$ systems attributed to the antibacterial activities [3]. In addition, functionalized polymer dots have substantially higher photostability and biocompatibility than fluorescent organic dyes and QDs, broadening their potential bio-application in various domains. Nitrogen doping is vital for achieving higher UV-Vis absorption and quantum yield. CPDs with nitrogen functional groups on their surface, such as amide and amino groups, have more excellent solubility in water. As a result, CPDs doped with nitrogen have a higher quantum yield and an extensive fluorescence lifetime. The PL spectra and UV-Vis absorption are likewise affected $[10,16,46]$. This review aims to study the CPDs, their photoluminescence properties, their dependence on nitrogen doping to enhance the photoluminescence properties as well as favoring the antibacterial studies

\section{Nitrogen-Doped CPDs}

Several synthesis methods have experimented with for the formation of CPDs with varying structures that exhibit tunable properties, which are useful for many specific applications. Control over the structure of CPDs' is the main objective to alter their properties for specific use. The CPDs are normally synthesized by bottom-up approaches such as microwave assistant hydrothermal, microwave assistant solvothermal, one-step hydrothermal and solvothermal, and or solvothermal, etc. In the "bottom-up" method, the CPDs go through additional carbonization and dehydration processes. The composition of CPDs can vary from amorphous to graphitic core based on the temperature assisted for the synthesis route. For temperature above $300^{\circ} \mathrm{C}$ leads to major graphitization, while cooler reactions outcome as amorphous particles. Thus, CPDs attained from "bottom-up" -methods have polymeric structures or polymer/carbon hybrid structures. As reported by Lu et al. [17], with the increase in temperature for synthesis, the structure of CPDs developed from a cross-linked polymer cluster (prepared at $100{ }^{\circ} \mathrm{C}$ ) via amorphous carbon structure (sample prepared at 200C) to 
carbon lattice (sample prepared at $300{ }^{\circ} \mathrm{C}$ ). The Photo Luminescence Quantum Yield (PLQY), presence of graphitic nitrogen and carbon lattice, increased with temperature from sample CPDs-100 to CPDs-300 [17].

Red emissive CPDs were synthesized by Liu et al. [6] from o-phenylenediamine by the addition of nitric acid $\left(\mathrm{HNO}_{3}\right)$, assisting the one-step hydrothermal method. Nitric acid acted as a catalyst in this reaction, and it also affected the structure of CPDs by oxidative polymerization, nitration, reduction, carbonization reactions, and protonation [6]. The doping strategy is mainly adopted to pertain to its unique features, thus making the CPDs multifunctional. Recent researches have shown that as the nitrogen content increases, both the emission and absorption peaks of nitrogen-doped CDs shift to longer wavelengths, and also, its electronic properties fluctuate significantly from those of the corresponding non-doped CDs. Experiments revealed that graphitic $\mathrm{N}$ centers induce a strong redshift in the CDs' absorption spectra due to the electron-doping effect, which decreases the scale of the electronic gap while doping with amino $\mathrm{N}$ centers, pyrrolic and pyridinic failed to show remarkable redshifts, which was attributed to the lack of electron doping [18].

In the hydrothermal method, these CPDs are synthesized by dissolving $0.5 \mathrm{mmol}$ of precursor in $10 \mathrm{ml}$ of deionized water and adding a nitrogen group with constant stirring. The prepared mixture is heated in the furnace at $200^{\circ} \mathrm{C}$ for 200 hours and then filtered through the membrane, dialyzed, and dried. In the case of the microwave-assisted pyrolysis method, the nitrogen group is dissolved in $10 \mathrm{ml}$ of ultrapure water, and the precursor is added to it. Then $5 \mathrm{ml}$ of distilled water is added into the mixture, followed by heating in the oven for about 1 min. This process is continued till the color changes and the gel is well distributed in distilled water. This solution is then centrifuged at $15000 \mathrm{rmp}$ for $10 \mathrm{~min}$, filtered, and dialyzed.

\section{Photoluminescence of CPDs}

CPDs have wide possible applications in the fields of bio-imaging and fluorescence sensors. The photoluminescence mechanism exhibited by CD's is a hot topic of discussion within the research community. The photoluminescence of CPDs originates mainly from the subdomain state, surface state, carbon core, molecular state, and the cross-link enhanced emission (CEE) effect. The higher quantum yield of CPDs, when compared to fully carbonized $\mathrm{CDs}$, is due to this photoluminescence mechanism. Photoluminescence of CPDs depends mainly on temperature, reaction time, $\mathrm{pH}$, carbonization degree, and doping. It was reported that the photoluminescence quantum yield (PLQY) increases with the increase in temperature. The higher synthesis temperature results in effective graphitization as well as a higher carbonization degree, which results in a larger conjugation structure and narrow bandgap with possible redshift emission. It was observed that a shift in emission wavelength and change in photoluminescence intensity occurred depending on the carbonization temperature. In other words, stronger photoluminescence was observed for CDs that are synthesized with a longer carbonization time. [6]

The four universally accepted photoluminescence strategies are (i) molecular state luminescence (ii) Cross-link enhanced emission effect (CEE), (iii) carbon core state luminescence, and (iv) surface state luminescence. The cross-linking of CPDs can confine vibration and rotation, increasing radiative transition probably to initiate improved fluorescence [6]. Figure $1(a, b)$ shows the cross-linking of CPDs includes a covalently crosslinked framework, intraparticle hydrogen bonds, physical cross-linked structures, and luminescence centers. Carbonization enhances the formation of rigid structures, which leads to 
physical cross-linking points to generate CEE to enhance emission Figure 1 (d). Crosslinking can control the rotation and vibration to decrease the non-radiation, causing an increase in the radiative transition probability to enhanced fluorescence Figure1 $(a, b)$. The rotation and vibration can also be restricted by lowering the temperature, thus enhancing photoluminescence intensity Figure1 (c). The cross-linking comprises intraparticle hydrogen bonds, coupled luminescence centers, physical-cross-linked structures, covalently crosslinked frameworks, etc. As a result, by increasing the carbonization degree, photoluminescence can be improved $[2,7,18-20]$.

(a)

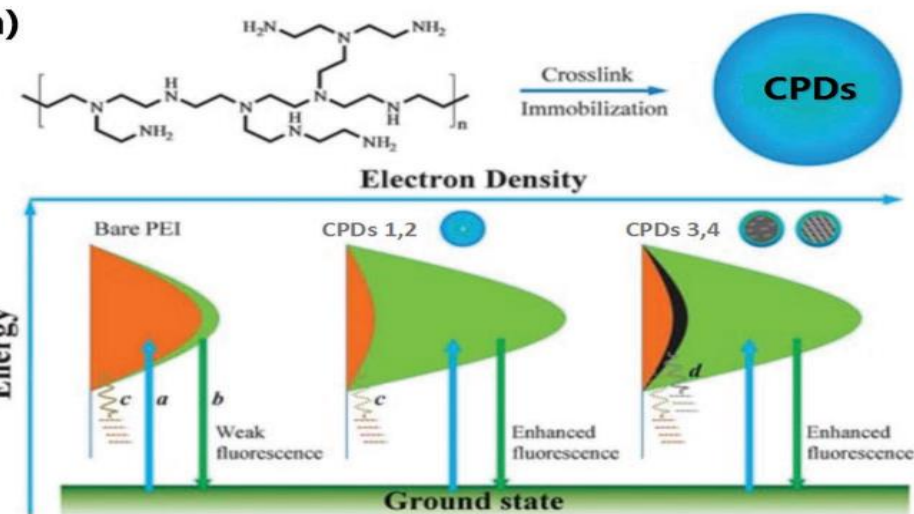

(b)

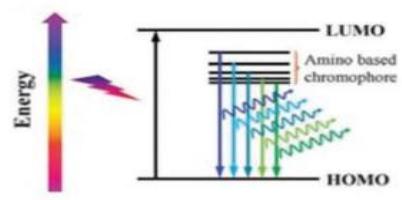

(c)

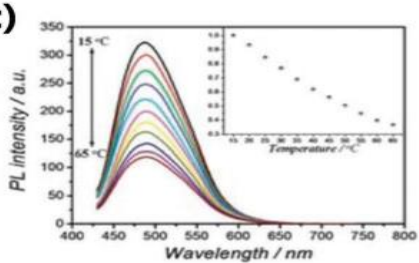

(d)

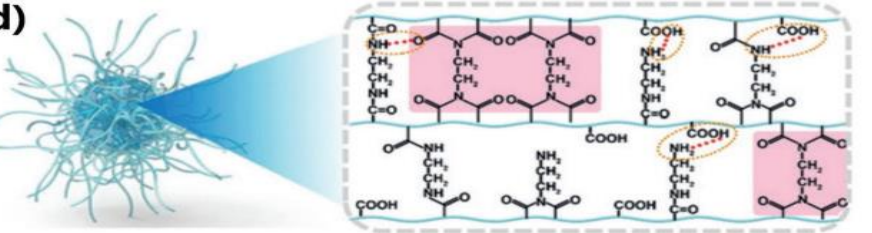

Crosslink Enhanced Emission Effect:

1) covalently-crosslinked frameworks

2) coupled luminescence centers

3) intraparticle hydrogen bonds

4) physical-crosslinked structures

Figure 1. Diagram for photoluminescence mechanism of bare PEI and CPD's. (a)Electrons trapped by amino acids after getting excited from the ground state (b) ground state recoiling of electrons through radiative route

(c) ground state recoiling of electrons through vibration and rotation non-radiative route; (d) ground state recoiling of electrons through a carbon-based non-radiative route [10] (Reprinted with permission).

\section{Nitrogen Doping and Red-Shift in CPDs}

Nitrogen-doped carbonized materials are finding applications in various fields due to their ability to exhibit fluorescence, thereby being used as biological markers [21]. Fluorescent materials have wide applications in fluorescence detection, drug delivery, and life marker diagnosis. After discovering CPDs, it was acknowledged as the new class of fluorescent material showing superior characteristics such as good biocompatibility, less toxicity, high light stability, and bleaching stability. Easy availability of raw materials and lesser production cost make it even more attractive from a research perspective [22-25].

The common elements used for doping includes nitrogen [26, 27], phosphorus [28], sulphur [29], boron [28, 30], some metals [31, 32] etc. Along with enhancing the photoluminescence of CPDs, Nitrogen doping can also be used to control the photoluminescence wavelength [6]. The most common types of nitrogen doping found are pyrrolic, graphitic, pyridinic, and amino centers (Figure 2) [33-45]. These groups are more likely to experience the transition to the lowest excited singlet state from the ground state of the electron, enhancing the surface state-related luminescence characteristics of CPDs significantly. The emission/absorption wavelength of CPDs can be redshifted to longer wavelength ranges through doping $\mathrm{N}$-containing groups. The charge transfer from the nitrogen group to the core is responsible for the rise in redshift as the functionalization degree of the 
CPDs increases. At the same time, excitation dependence can be attributed to different molecular states and the occurrence of distinct types of nitrogen subunits on the surface. Furthermore, with increased graphitic N concentration, a significant PL redshift was detected. [46-50] The redshift emission in CPDs' may be induced by nitrogen-containing fluorophores, improving the fluorescence behavior. Crosslinking improves fluorescence by limiting vibration and rotation, lowering the non-radiation, and improving the possibility of radiative transition, all of which contribute to more significant fluorescence. The edge $\mathrm{N}$-containing functional groups (graphitic-N) might control the energy level and improve the radiation transition possibility, raising PL intensity by increasing the domains' rigidity and reducing non-radiative relaxation caused by molecular vibrations on the CPDs' surface [6, 51-55].

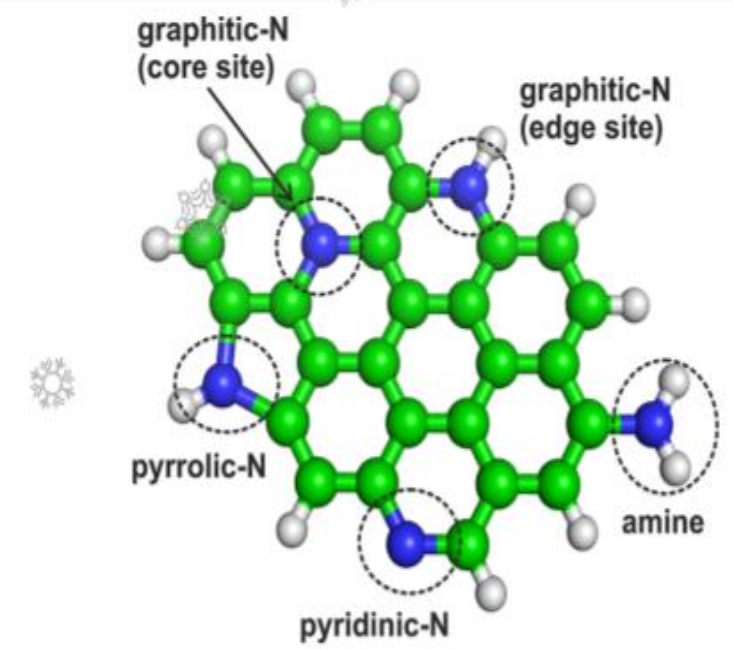

Figure 2. Common types of N-doping [33] (Reprinted with permission).

Recent experiments showed that the emission and absorption peaks of both nitrogendoped Carbon Dots (CDs) and CPDs shift to longer wavelengths with increasing nitrogen content $[33,34,37]$. According to the studies, the graphitic $\mathrm{N}$ doping caused a redshift of their absorption spectra in CDs. Still, other nitrogen configurations like pyrrolic and pyridinic doping showed no such effects $[3,36]$. At the same time, it was reported by JunJun Liu et al. [3] that due to nitrogen doping, Pyrrolic $\mathrm{N}$ and the Pyridinic N, contributed to form Carbonized Polymer Dots (CPDs) [3, 38]. Nitrogen doping decreases the lowest unoccupied molecular orbital and the highest occupied molecular orbital (HOMO-LUMO) gap, reducing energies of corresponding optical transitions, i.e., they can pump up extra electrons to the unoccupied $\pi^{*}$ orbitals of the carbon [36]. As formed, nitrogen-doped CPDs possess more efficient conjugated aromatic $\pi$ with a pair of p-electrons which contributes to the intense red luminescence. JunJun Liu et al. [3] synthesized red emissive CPDs from o-phenylenediamine (oPD) by nitrogen doping and found that it showed strong red luminescence with a high dosage of $\mathrm{HNO}_{3}$. Nitrogen doping led to increased size, hydrogen bonds, and more efficient conjugated aromatic $\pi$ systems that played an essential part in the optical characteristics of CPDs. They resulted in the redshift of absorption and emission wavelength. All these characteristics of CPDs can be regulated by altering the quantity of $\mathrm{HNO}_{3}$. The red emissive CPDs were an excellent fluorescence probe for bioimaging, both in vivo and in vitro [3]. Jie Xia et al. [34] proposed a one-pot low temperature aqueous synthetic route for highly stable red emissive CPDs (R-CPDs). The PL mechanism of the R-CPDs was studied by investigating the chemical composition, morphology, and photophysical properties. The R-CPDs exhibited a broad color-tunable range of solvent-dependent emission, high $\mathrm{pH}$ sensitivity, and good resistance to high salinity and 
photobleaching, high colloidal, optical stability in physiological media as well as low cytotoxicity [34].

The luminescent carbon dots with higher fluorescence quantum yield were synthesized from ammonium citrate and triethylenetetramine through microwave-assisted pyrolysis. Comparing CDs, doped with nitrogen, synthesized at various reaction times shows that quantum yield is almost the same for other reaction times. Also, the doped carbon dots prepared at high power showed a higher quantum yield than those prepared at lower power. The PL spectrum recorded at different concentration ratios of the reactant indicates that the quantum yield is maximum when the quantity of substances is equal (Figure 3). The experiment was conducted for different ratios, among which similar ratios showed the highest quantum yield. When the synthesis of doped carbon dots was done through one step and poly step reactions, the poly step reaction showed high quantum yield than the other [21].
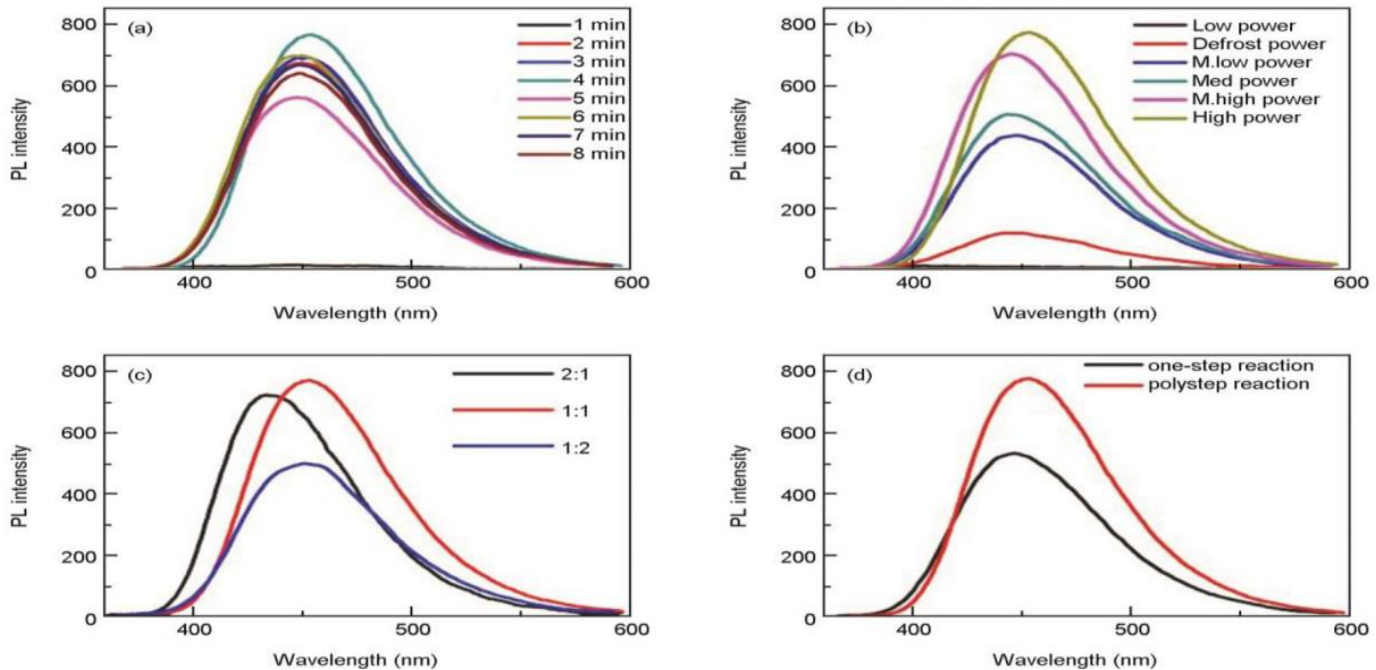

Figure 3. PL spectrum showing (a) N-CDs synthesized at various reaction times; (b) N-CDs synthesized at varying powers; (c) different molar ratios of ammonium citrate to triethylenetetraine; (d) N-CDs synthesized via various reaction methods $\left(\lambda_{\mathrm{ex}}=360 \mathrm{~nm}\right)$ with concentration of $\mathrm{N}-\mathrm{CDs}=0.2 \mathrm{mg} / \mathrm{mL}$ [21] (Reprinted with permission).
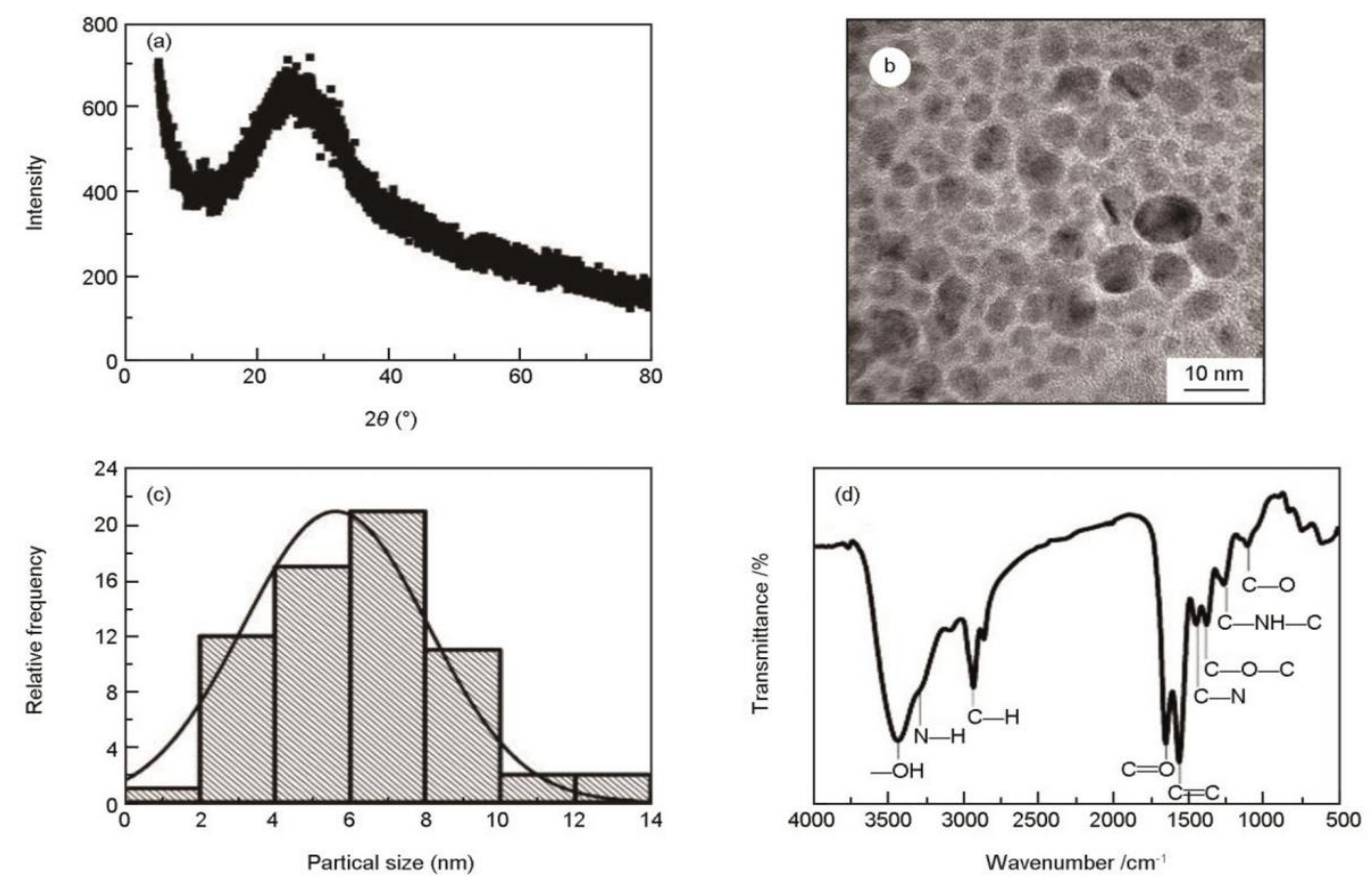

Figure 4. (a) XRD pattern of the N-CDs; (b) TEM image of the N-CDs, scale bar is $10 \mathrm{~nm}$; (c) the diameter distribution of the N-CDs; (d) FTIR spectrum of the N-CDs [21] (Reprinted with permission). 
The XRD and FTIR spectrum of the nitrogen-doped CPDs were taken and analyzed. In Fig. 4 (a), a broad diffraction peak at $2 \theta$ value at 25 degrees, giving the idea that the N-CPD's are amorphous. The TEM image of the sample shown in Figure 4 (b) suggests that the N-CPD's are spherical in their morphology. Figure 4 (c) displays the particle size distribution of the nitrogen-doped carbon dots. It shows that the particle size ranges around 6-8 nanometers. Fig. 4 (d) shows the FTIR spectrum of doped carbon dots. The functional groups were identified by the broad absorption bands. The functional groups were determined to be $\mathrm{O}-\mathrm{H}, \mathrm{N}-\mathrm{H}$, and $\mathrm{C}=\mathrm{O}$ at $3420 \mathrm{~cm}^{-1}, 3274 \mathrm{~cm}^{-1}$ and $1108 \mathrm{~cm}^{-1}$ respectively. This result shows that the nitrogen-doped carbon dots consist of Nitrogen and Oxygen functional groups, indicating excellent water solubility favorable for additional modifications and thus accounting for various applications [21]. The PL properties (bright red fluorescence) and functional groups consisting of N-and/or $\mathrm{O}$ and the cross-link enhanced emission (CEE) effect have a close relationship [13, 39].

The studies reveal that nitrogen increases the Fermi level, which enriches electrons and reduces surface defects, thereby enhancing the PL quantum yield (QY) [13]. The amount of nitrogen doping affects the shift in Fermi energy. Nitrogen doping creates states in the energy gap between the primary position of Fermi energy and the CBs and VBs, separately. That means the doping with nitrogen-containing group causes impurity levels to rise below the CBs. As a result, the Fermi level decreases and tends toward lower energy. [47] This change becomes more pronounced as doping concentration increases. Transitions from HOMO to LUMO of the functional subunits determine the shift in peak position. However, the addition of the nitrogen group does not affect functional subunits, a lone pair electron of $\mathrm{N}$-atom conjugates with the pi network of the parent structure without altering the HOMO and LUMO's planar symmetry. Therefore, the $2 \mathrm{p}_{\mathrm{z}}$ orbital of $\mathrm{N}$-atom makes a significant contribution to the HOMO. A charge transmission is observed from the nitrogen group to its parent structure along with HOMO to LUMO transition. The pronounced redshift is due to this charge transfer [48]. Due to the abundant valence electron present on the nitrogen atom, graphitic dopant causes an excess charge distribution in the carbon pi state. The presence of graphitic nitrogen in the structure narrows the HOMO-LUMO gap, triggering the redshift. According to calculations, the presence of graphitic $\mathrm{N}$ centers causes a significant redshift in CDs' absorption spectra, reducing the magnitude of the electronic gap due to an electron-doping effect. To mention that there were no appreciable redshifts seen when pyridinic, pyrrolic, or amino $\mathrm{N}$ centers were doped, proving the lack of electron doping. [49,50] Hence CPDs include several complex energy levels as well as fluorescence centers, significantly enhancing photoluminescence.

\section{Applications of CPDs}

Recent years are witnessing the emergence of CPDs with a higher range of carbon content as a green and environmentally benign material. Due to their unique property, they find several applications. These features find applications in the construction of optoelectronic devices, catalysis, ion detection, etc. CPDs own exceptional biocompatibility, water dispersibility, strong penetrability, and high QY; hence, they have many biomedical applications [3].

Liu et al. [3] reported the synthesis of red emissive CPDs, as an excellent fluorescence agent favoring both in vitro and in vivo bioimaging. For the biodistribution imaging studies, $100 \mu \mathrm{L}$ of CPDs was injected into mice through its vein, and the fluorescence images were collected at specific time intervals. $0.5 \mathrm{~h}$ post-injection, clear and strong fluorescence intensity was observed in the mice's whole body. As the time increased, a gradual reduction in the signal 
intensity of the fluorescence signal was observed. After $20 \mathrm{~h}$ of circulation, the signal turns out to be very weak. Optimal bioimaging was observed around $0.5 \mathrm{~h}$ post-injection. The injected CPDs entered the whole body of the mice along with the circulating blood and could be quickly oozed from the mice's bodies [3].

Phosphorescence is the emission of radiation similar to fluorescence emission but on a longer timescale. So, the emission continues even after the excitation ceases. But the phosphorescence action is reduced in effect due to the spin-forbidden property of triplet exciton transition and non-radiative decay. Very low temperatures are essential to get operational phosphorescence. Various methods have been implemented to achieve room-temperature phosphorescence (RTP), which is the phosphorescence emission achievement at room temperatures. Many attempts are going on and have already been reported in search of RTP materials. These materials can be classified as those depending on the use of organometallic complexes and those concentrating on clean RTP materials (generally using platinum, iridium, and various noble metals). The CPDs with large, highly cross-linked structures of nonconjugated groups can achieve the metal-free RTP assisting suitable synthesis methods. CPDs holds the properties of both polymers and carbon dots, i.e., possess excellent luminescent properties of carbon dots and the polymers' matrix effect [10].

Since we can control CPDs' structure, it's possible to alter their features for specific applications. It was observed that, subjected to the synthesis temperature, the CPDs' core's configuration can lead to graphitic from amorphous. There are reports of synthesis of several types of CPDs with methodical structure alteration through a uniform amino acid reaction. The increase in synthesis temperature observed a structure developed from a cross-linked polymer cluster to a carbon lattice. The sample CPDs300 (synthesized at $300^{\circ} \mathrm{C}$ ) showed a welldeveloped carbon lattice, graphitic nitrogen's increased presence, and the highest PLQY among the three samples investigated. The as-prepared carbon dots also showed room-temperature ferromagnetism. This work could open another space of utilization of carbon dots and perhaps lead to carbon-based spintronic gadgets working under surrounding conditions [17].

Many of the CPDs can still not be used for their wide range of applications due to their lesser QY and self-quenching. The low $Q Y$ is due to sub fluorophores $(C=N, C=O, N=O)$, resulting in very little photoluminescence. Crosslinking was used to enhance photoluminescence, and cross-linking enhanced emission effect (CEE effect) was put forward through this process [40].

Figure 5 shows that the oxygen-rich precursors can be used for the synthesis of CPDs. It was found that $\mathrm{CO}_{2}$ also could be used as a precursor for the preparation of CPDs. CPDs were prepared through the solvothermal method of EDA and TPTE. Also, the CPDs were applied as single phosphor, which may reduce reabsorption, color imbalance, etc. The formation of CPDs consuming polymers as the precursor material experiences cross-linking, carbonization and dehydration, and the CEE effect can increase the QY of CPDs. Strong blue luminescence exhibited the excitation-dependent PL characteristics. Also, CPDs exhibit solidstate luminescence. [40] 


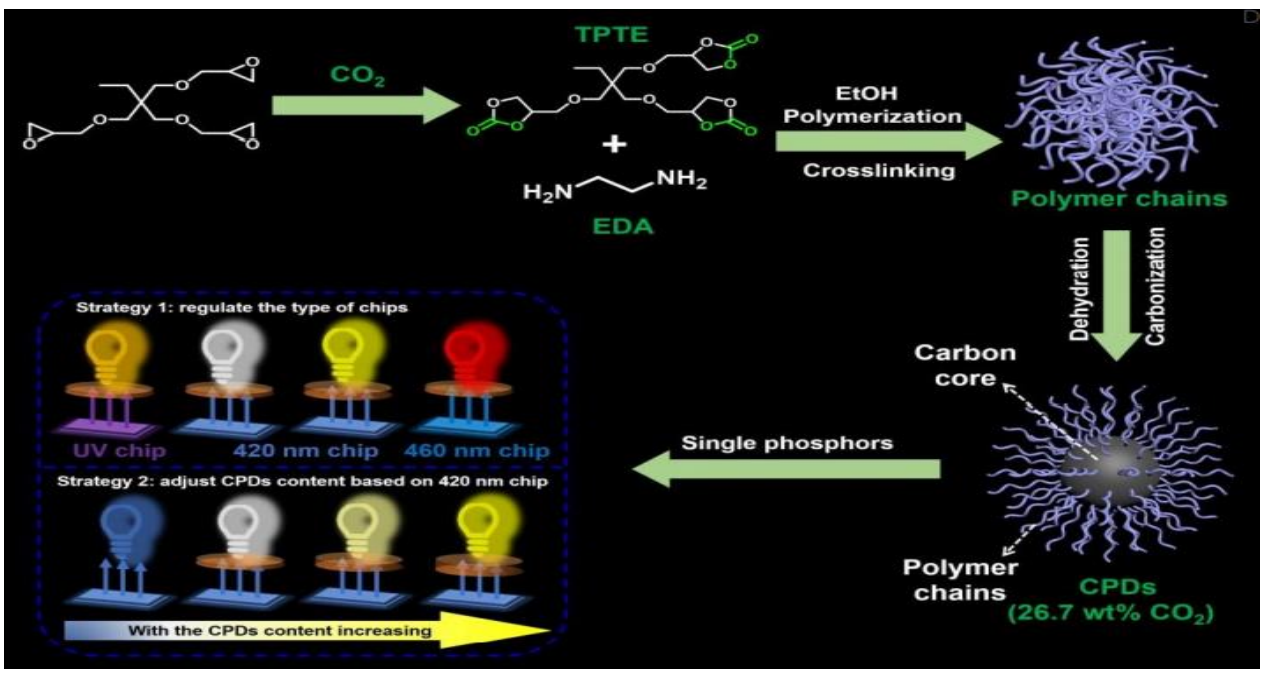

Figure 5. Formation of CPDs and their application in multicolour LEDs [32] (Reprinted with permission).

Due to its aggregation-triggered quenching phenomenon, most CPDs do not show fluorescence in solid-state and poor solvents. The blue to the red spectral composition of solidstate CPDs can range up to $69.4 \%$, evidently higher than when in solution form (45.6\%). This higher value helps in converting UV light into visible light, which in turn gives the possibility for its application in phosphor-converted LEDs. The CPDs were used as individual phosphors to design multicolor phosphor LEDs due to their high blue to red spectral composition, broad conversion peak, and direct solid-state luminescence [40].

Alzheimer's disease (AD), a protracted neurodegenerative disease, is triggered due to the fibrillization and accumulation of amyloid $\beta$-protein $(A \beta)$ in patients' brains. Gao et al. [1], in their work, found multifunctional $\mathrm{N}$-doped carbonized polymer dots for pointing $\mathrm{A} \beta$ aggregation, as well as toxicity. The studies on this revealed that CPDs could effectively modulate $A \beta$ aggregation, figure out $A \beta$ mature fibrils in a very short span of time, and generate off-pathway $\mathrm{A} \beta$ types via electrostatic interactions, hydrophobic interactions, and $\mathrm{H}$-bonding. From in vivo assays with $C$. elegans CL2006, the A $\beta$ searching and identifying competencies of CPDs was proven. This can lead to promising applications of carbon nanomaterials in the detection and recovery from neurodegenerative disorders as well as other protein conformational diseases.

\section{Conclusions}

The lack of a suitable bandgap in macro-sized carbon material is a drawback for it to be used as a luminescent material. In recent years, fluorescence carbon dots have drawn great attraction due to their superior properties like good fluorescence, excellent biocompatibility as well as low toxicity, etc. Many processes were established for synthesizing polymerized carbon dots. Through the process of carbonization of polymer, we can achieve superior properties to the individual materials. Among all the methods hydrothermal method is more advantageous of being simple, low cost, and environment friendly. The polymer undergoes several complicated alterations during the hydrothermal synthesis process, the most notable of which is cross-linking polymerization. Cross-linking occurs inside the polymer cluster as a result of this polymerization, making the polymer more compact and stable. The degree of carbonization rises during the hydrothermal process. As a result, the polymer's photoluminescence intensity is considerably reduced. The temperature, as well as the response time, is essential factors in the creation of CPDs. The properties of CPDs were altered by doping them with suitable 
dopants. It was observed that superior quantum yield was obtained due to doping. Doping CPDs with hetero-atoms such as phosphorous or nitrogen could further increase the fluorescence intensity and quantum yield.

CPDs are a novel type of CD that has a unique polymer/carbon hybrid structure and property. This study aims to provide a comprehensive knowledge of the connection between the absorption properties of N-doped CPDs and data that can aid in the design and synthesis of CPDs with better optical features. Despite substantial advances in CPD research, the introduction of bottom-up synthesis techniques has made it possible to synthesize many CPDs with various unique features. However, all of the CPDs have a significant short-wavelength emission flaw, which severely restricts their bio-applications. As a result, developing a suitable method for creating red or even near-infrared emission CPDs and revealing their photoluminescence (PL) processes remains a problem and an essential subject. Future studies should concentrate on the synthesis principle, how synthesis affects structure and performance, and a thorough knowledge of the PL and formation method. To date, CPD synthesis has been challenging to manage structural and performance regulation, severely limiting CPD applications such as tuning fluorescence wavelength in LEDs, CPDs with aiming functions for controllable surface groups for sensing, bio labeling, and biotherapy, and so on. In addition, the mechanism of the reaction, the nucleation mechanism, and the formation process are all unknown. Furthermore, due to the problematic study on complicated carbon/polymer hybrid structure and the non-availability of systematic structure characteristic measurements to show this structural feature, the precise structures of CPDs are yet unknown. All of these things need a great deal of investigation. Nevertheless, they pose a significant research challenge and opportunity in photovoltaic devices, biomedicine, sensing, light-emitting diode, anticounterfeiting, photocatalysis, etc.

\section{Funding}

This research was funded by the Department of Science and Technology, Ministry of Science and Technology, India, grant number DST/TMD/CERI/RES/2037(G).

\section{Acknowledgments}

This research was further supported by the Centre for Research, CHRIST (Deemed to be University), Bengaluru, India.

\section{Conflicts of Interest}

The authors declare no conflict of interest.

\section{References}

1. Wang, C.; Li, L.; Wu, Y.; Gu, J.; Ma, C.; Zhu, C.; Gao, H.; Zhang, Y.; Shang, Y.; Yang, Z.; Chen, G. Highly Photoluminescent Carbon Dots with pH-Dependent Switchable Fluorescence and Sensitivity to Tetracycline. Nano. 2021, 03, 2150036, https://doi.org/10.1142/S1793292021500363.

2. Liu, J.; Lu, S.; Tang, Q.; Zhang, K.; Yu, W.; Sun, H.; Yang, B. One-step hydrothermal synthesis of photoluminescent carbon nanodots with selective antibacterial activity against Porphyromonas gingivalis. Nanoscale 2017, 21, 7135-7142, https://doi.org/10.1039/C7NR02128C.

3. Liu, J.; Li, D.; Zhang, K.; Yang, M.; Sun, H.; Yang, B. One-step hydrothermal synthesis of nitrogen-doped conjugated carbonized polymer dots with $31 \%$ efficient red emission for in vivo imaging. Small. 2018, 15, 1703919, https://doi.org/10.1002/smll.201703919. 
4. Zhang, X.; Jiang, M.; Niu, N.; Chen, Z.; Li, S.; Liu, S.; Li, J. Natural-product-derived carbon dots: from natural products to functional materials. ChemSusChem. 2018, 11, 11-24, https://doi.org/10.1002/cssc.201701847.

5. Roy, P.; Chen, P.C.; Periasamy, A.P.; Chen, Y.N.; Chang, H.T. Photoluminescent carbon nanodots: synthesis, physicochemical properties and analytical applications. Materials Today. 2018, 8 , 447-458, https://doi.org/10.1016/j.mattod.2015.04.005.

6. Xia, C.; Zhu, S.; Feng, T.; Yang, M.; Yang, B. Evolution and synthesis of carbon dots: from carbon dots to carbonized polymer dots. Advanced Science. 2019, 23, 1901316, https://doi.org/10.1002/advs.201901316.

7. Xia, C.; Tao, S.; Zhu, S.; Song, Y.; Feng, T.; Zeng, Q.; Liu, J.; Yang, B. Hydrothermal Addition Polymerization for Ultrahigh-Yield Carbonized Polymer Dots with Room Temperature Phosphorescence via Nanocomposite. Chemistry-A European Journal. 2018, 44, 11303-11308, https://doi.org/10.1002/chem.201802712.

8. Song, Y.; Zhu, S.; Shao, J.; Yang, B. Polymer carbon dots-a highlight reviewing their unique structure, bright emission and probable photoluminescence mechanism. Journal of Polymer Science Part A: Polymer Chemistry. 2017, 4, 610-615, https://doi.org/10.1002/pola.28416.

9. Song, Y.; Zhu, S.; Zhang, S.; Fu, Y.; Wang, L.; Zhao, X.; Yang, B. Investigation from chemical structure to photoluminescent mechanism: a type of carbon dots from the pyrolysis of citric acid and an amine. Journal of Materials Chemistry C. 2015, 23, 5976-5984, https://doi.org/10.1039/C5TC00813A.

10. Tao, S.; Lu, S.; Geng, Y.; Zhu, S.; Redfern, S.A.; Song, Y.; Feng, T.; Xu, W.; Yang, B. Design of metal-free polymer carbon dots: a new class of room-temperature phosphorescent materials. Angewandte Chemie International Edition. 2018, 9, 2393-2398, https://doi.org/10.1002/anie.201712662.

11. Miao, X.; Qu, D.; Yang, D.; Nie, B.; Zhao, Y.; Fan, H.; Sun, Z. Synthesis of carbon dots with multiple color emission by controlled graphitization and surface functionalization. Advanced materials. 2018, 1, 1704-740, https://doi.org/10.1002/adma.201704740.

12. Zheng, K.; Li, X.; Chen, M.; Gong, Y.; Tang, A.; Wang, Z.; Wei, Z.; Guan, L.; Teng, F. Controllable synthesis highly efficient red, yellow and blue carbon nanodots for photo-luminescent light-emitting devices. Chemical Engineering Journal. 2020. 122503, https://doi.org/10.1016/j.cej.2019.122503.

13. Tao, S.; Song, Y.; Zhu, S.; Shao, J.; Yang, B. A new type of polymer carbon dots with high quantum yield: from synthesis to investigation on fluorescence mechanism. Polymer 2017, 472-478.

14. Tao, S.; Song, Y.; Zhu, S.; Shao, J.; Yang, B. Beyond bottom-up carbon nanodots: Citric-acid derived organic molecules. Nano Today. 2016, 2 , 128-132, https://doi.org/10.1016/j.polymer.2017.02.039.

15. Tao, S.; Feng, T.; Zheng, C.; Zhu, S.; Yang, B. Carbonized Polymer Dots: A Brand New Perspective to Recognize Luminescent Carbon-Based Nanomaterials. The Journal of Physical Chemistry Letters 2019, 17, 5182-5188, https://doi.org/10.1021/acs.jpclett.9b01384.

16. Tao, S.; Zhu, S.; Feng, T.; Xia, C.; Song, Y.; Yang, B. The polymeric characteristics and photoluminescence mechanism in polymer carbon dots: A review. Materials today chemistry. 2017, 13-25, https://doi.org/10.1016/j.mtchem.2017.09.001.

17. Ding, H.; Li, X.H.; Chen, X.B.; Wei, J.S.; Li, X.B.; Xiong, H.M. Surface states of carbon dots and their influences on luminescence. Journal of Applied Physics 2020, 23, 231101, https://doi.org/10.1063/1.5143819.

18. Gaur, A.; Rana, D.; Maiti, P. Mechanical and wear behaviour of poly (vinylidene fluoride)/clay nanocomposite. Journal of Materials Research and Technology 2019, 8, 5874-81, https://doi.org/10.1016/j.jmrt.2019.09.059.

19. Guo, L.; Ge, J.; Wang, P. Pengfei, Wang. Polymer dots as effective phototheranostic agents. Photochemistry and photobiology. 2018, 5 , 916-934, https://doi.org/10.1111/php.12956.

20. Gao, W.; Wang, W.; Dong, X.; Sun, Y. Nitrogen-Doped Carbonized Polymer Dots: A Potent Scavenger and Detector Targeting Alzheimer's $\quad \beta$-Amyloid Plaques. Small. 2020, 43, 2002804, https://doi.org/10.1002/smll.202002804.

21. Liu, W.; Zhang, R.; Kang, Y.; Zhang, X.Y.; Wang, H.J.; Li, L.H.; Diao, H.P; Wei, W.L. Preparation of nitrogen-doped carbon dots with a high fluorescence quantum yield for the highly sensitive detection of $\mathrm{Cu} 2+$ ions, drawing anti-counterfeit patterns and imaging live cells. New Carbon Materials. 2019, 4, 390-402, https://doi.org/10.1016/S1872-5805(19)30024-1.

22. Lim, S.Y.; Shen, W.; Gao, Z. Carbon quantum dots and their applications. Chemical Society Reviews. 2015, $1,362-381,10.1039 / \mathrm{C} 4 \mathrm{CS} 00269 \mathrm{E}$. 
23. Ramya, A.V.; Balachandran, M. Valorization of agro-industrial fruit peel waste to fluorescent nanocarbon sensor: Ultrasensitive detection of potentially hazardous tropane alkaloid. Front. Environ. Sci. Eng. 2021, 16, 1-11, https://doi.org/10.1007/s11783-021-1461-z.

24. Adrita, S.H.; Tasnim, K.N.; Ryu, J.H.; Sharker, S.M. Nanotheranostic Carbon Dots as an Emerging Platform for Cancer Therapy. Journal of Nanotheranostics. 2020, 1, 58-77, https://doi.org/10.3390/jnt1010006.

25. Yu, S.P.; Su, X.D.; Du, J.L.; Wang, J.L.; Gao, Y.D.; Zhang, L.; Chen, L.; Yang, Y.Z.; Liu, X.G. The cytotoxicity of water-soluble carbon nanotubes on human embryonic kidney and liver cancer cells. New Carbon Materials. 2018, 33, 36-45, https://doi.org/10.1016/S1872-5805(18)60325-7.

26. Diao, H.; Li, T.; Zhang, R.; Kang, Y.; Liu, W.; Cui, Y.; Wei, S.; Wang, N.; Li, L.; Wang, H.; Niu, W. Facile and green synthesis of fluorescent carbon dots with tunable emission for sensors and cells imaging[J]. Spectrochimica Acta Part A: Molecular and Biomolecular Spectroscopy. 2018, 200, 226-234, https://doi.org/10.1016/j.saa.2018.04.029.

27. Bai, Y.; Zhao, J.; Wang, S.; Lin, T.; Ye, F.; Zhao, S. Carbon Dots with Absorption Red-Shifting for TwoPhoton Fluorescence Imaging of Tumor Tissue $\mathrm{pH}$ and Synergistic Phototherapy. ACS Applied Materials \& Interfaces. 2021, https://doi.org/10.1021/acsami.1c08076.

28. Chen, X.; Jin, Q.; Wu, L.; Tung, C.; Tang, X. Synthesis and Unique Photoluminescence Properties of Nitrogen-Rich Quantum Dots and Their Applications. Angew. Chem., Int. Ed. 2014, 53, 12542- 12547, https://doi.org/10.1002/ange.201408422.

29. Daniel, S.; KS, S. Highly luminescent biocompatible doped nano carbon dot composites as efficient antibacterial agents. Composite Interfaces. 2021, 1-16, https://doi.org/10.1080/09276440.2020.1867466.

30. Coros, Maria.; Codruta, Varodi.; Florina, Pogacean.; Emese, Gal.; Stela M, Pruneanu. Nitrogen-doped graphene: The influence of doping level on the charge-transfer resistance and apparent heterogeneous electron transfer rate. Sensors. 2020, 7, 1815, https://doi.org/10.3390/s20071815.

31. Ramya, A.V.; Mohan, A.N.; Manoj, B. Wrinkled graphene: synthesis and characterization of few layer graphene-like nanocarbons from kerosene. Material Science-Poland. 2016, 2, 330-336, https://doi.org/10.1515/msp-2016-0061.

32. Liu, B.; Chu, B.; Wang, Y.L.; Hu, L.F.; Hu, S.; Zhang, X.H. Carbon dioxide derived carbonized polymer dots for multicolor light-emitting diodes. Green Chemistry. 2021, 23, 422-429, https://doi.org/10.1039/D0GC03333B.

33. Meng, W.; Wang, B.; Ai, L.; Song, H.; Lu, S. Engineering white light-emitting diodes with high color rendering index from biomass carbonized polymer dots. Journal of Colloid and Interface Science 2021, 598, 274-282, https://doi.org/10.1016/j.jcis.2021.04.022.

34. Xia, J.; Chen, S.; Zou, G.Y.; Yu, Y.L.; Wang, J.H. Synthesis of highly stable red-emissive carbon polymer dots by modulated polymerization: from the mechanism to application in intracellular $\mathrm{pH}$ imaging. Nanoscale 2018, 47, 22484-22492, https://doi.org/10.1039/C8NR08208A.

35. Tadesse, A.; Hagos, M.; RamaDevi, D.; Basavaiah, K.; Belachew, N. Fluorescent-nitrogen-doped carbon quantum dots derived from citrus lemon juice: green synthesis, mercury (II) ion sensing, and live cell imaging. ACS omega. 2020, 8, 3889-3898, https://doi.org/10.1021/acsomega.9b03175.

36. Venkatesan, R.A.; Joseph, N.; Balachandran, M. Green Synthesis of Reduced Graphene Nanostructure from Cinnamomum Camphora. Letters in Applied NanoBioScience. 2020, 10, 2003-2011, https://doi.org/10.33263/LIANBS101.20032011.

37. Guggilla, P.; Kassu, A.; Simpson, D. Reducing the bandgap energy via doping process in lead-free thin film nanocomposites. Research and Reviews: Journal of Materials Science. 2017, 5, 34-44, https://doi.org/10.1117/12.2504000.

38. Zhang, Z.; Xiong, J.; He, G.; Dang, D.; Xie, Y.; Wang, Q. Fluorous Effect-Induced Emission of Azido Substituted Poly (vinylidene fluoride) with High Photostability and Film Formation. Polymer Chemistry. 2020, 11, 1307-1313, https://doi.org/10.1039/C9PY01622H.

39. Tian, J.; Zhang, J.; Yang, J.; Du, L.; Geng, H.; Cheng, Y. Conjugated polymers act synergistically with antibiotics to combat bacterial drug resistance. ACS applied materials \& interfaces. 2017, 9, 18512-18520, https://doi.org/10.1021/acsami.7b03906.

40. Tao, S.; Zhu, S.; Feng, T.; Zheng, C.; Yang, B. Crosslink-Enhanced Emission Effect on Luminescence in Polymers: Advances and Perspectives. Angewandte Chemie. 2020, 25, 9910-9924, https://doi.org/10.1002/ange.201916591.

41. Bagheri, N.; Lakouraj, M.M.; Hasantabar, V.; Mohseni, M. Biodegradable macro-porous CMC-polyaniline hydrogel: synthesis, characterization and study of microbial elimination and sorption capacity of dyes from 
waste water. Journal of Hazardous Materials. $\quad$ 2021, 403, $\quad$ 123631, https://doi.org/10.1016/j.jhazmat.2020.123631.

42. Bai, H.; Zhang, H.; Hu, R.; Chen, H.; Lv, F.; Liu, L.; Wang, S. Supramolecular conjugated polymer systems with controlled antibacterial activity. Langmuir 1116-1120, https://doi.org/10.1021/acs.langmuir.6b04469.

43. Mucha, S.G.; Firlej, L.; Bantignies, J.L.; Żak, A.; Samoć, M.; Matczyszyn, K. Acetone-derived luminescent polymer dots: a facile and low-cost synthesis leads to remarkable photophysical properties. RSC Advances. 2020, 63, 38437-38445, https://doi.org/10.1039/D0RA05957A.

44. Wang, C.; Zhou, J.; Ran, G.; Li, F.; Zhong, Z.; Song, Q.; Dong, Q. Bi-functional fluorescent polymer dots: a one-step synthesis via controlled hydrothermal treatment and application as probes for the detection of temperature and Fe 3+. Journal of Materials Chemistry 2017, 2, 434-443, https://doi.org/10.1039/C6TC04286D.

45. Thomas, R.; Balachandran, M. Luminescence and energy storage characteristics of coke-based graphite oxide. Materials Chemistry and Physics 2021, 257, https://doi.org/10.1016/j.matchemphys.2020.123854.

46. Lu, S.; Sui, L.; Wu, M.; Zhu, S.; Yong, X.; Yang, B. Graphitic Nitrogen and High-Crystalline Triggered Strong Photoluminescence and Room-Temperature Ferromagnetism in Carbonized Polymer Dots. Advanced Science. 2019, 2 ,1801192, https://doi.org/10.1002/advs.201801192.

47. Chen, Y.; Zheng, M.; Xiao, Y.; Dong, H.; Zhang, H.; Zhuang, J.; Hu, H.; Lei, B.; Liu, Y. A self-quenchingresistant carbon-dot powder with tunable solid-state fluorescence and construction of dual-fluorescence morphologies for white light-emission. Advanced Materials 2016, 2, 312-318, https://doi.org/10.1002/adma.201503380.

48. Carbonaro, C.M.; Corpino, R.; Salis, M.; Mocci, F.; Thakkar, S.V.; Olla, C.; Ricci, P.C. On the emission properties of carbon dots: reviewing data and discussing models. C-Journal of Carbon Research. 2019, 5, 60, https://doi.org/10.3390/c5040060.

49. Ramya, A.V.; Joseph, N.; Balachandran, M. Facile Synthesis of Few-Layer Graphene Oxide from Cinnamomum camphora. Nanobiotechnology $\quad$ Reports. $\quad \mathbf{2 0 2 1 ,} \quad 2, \quad$ 183-187, https://doi.org/10.1134/S2635167621020130.

50. Huang, W.; Li, X.; Sun, X.; Ding, X.; Feng, Y.; Tang, Y.; Zhou, P.; Wang, L.; Zhang, Q. Photoluminescence of graphene quantum dots enhanced by microwave post-treatment. Chemical Engineering Journal. 2021, 405, 126714, https://doi.org/10.1016/j.cej.2020.126714.

51. Janakiraman, S.; Surendran, A.; Ghosh, S.; Anandhan, S.; Venimadhav, A. Electroactive poly (vinylidene fluoride) fluoride separator for sodium ion battery with high coulombic efficiency. Solid State Ionic 2016, 292, 130-135, https://doi.org/10.1016/j.ssi.2016.05.020.

52. Li, F.; Wang, X.; Liu, W.; Wang, L.; Wang, G. One-step solvothermal synthesis of red emissive carbonized polymer dots for latent fingerprint imaging. Optical Materials. 2018, 86, 79-86, https://doi.org/10.1016/j.optmat.2018.09.040.

53. Aziz, S.B.; Brza, M.A.; Nofal, M.M.; Abdulwahid, R.T.; Hussen, S.A.; Hussein, A.M.; Karim, W.O. A comprehensive review on optical properties of polymer electrolytes and composites. Materials. 2020, 17, 3675, https://doi.org/10.3390/ma13173675.

54. Ding, H.; Li, X.H.; Chen, X.B.; Wei, J.S.; Li, X.B.; Xiong, H.M. Surface states of carbon dots and their influences on luminescence. Journal of Applied Physics. 2020, 23, 231101, https://doi.org/10.1063/1.5143819.

55. Li, F.; Wang, X.; Liu, W.; Wang, L.; Wang, G. One-step solvothermal synthesis of red emissive carbonized polymer dots for latent fingerprint imaging. Optical Materials. 2018, 86, 79-86, https://doi.org/10.1016/j.optmat.2018.09.040. 